\title{
Antihypertensive drug classes have different effects on short-term blood pressure variability in essential hypertension
}

\author{
Natacha Levi-Marpillat ${ }^{1,2,3}$, Isabelle Macquin-Mavier ${ }^{1,2}$, Anne-Isabelle Tropeano ${ }^{4}$, Gianfranco Parati ${ }^{5,6}$ \\ and Patrick Maison ${ }^{1,2,3}$
}

Increased blood pressure variability (BPV) contributes to end-organ damage, cardiovascular events and mortality associated with hypertension. In a cohort of $\mathbf{2 7 8 0}$ hypertensive patients treated by either calcium channel blockers (CCBs), diuretics, angiotensin-converting enzyme inhibitors (ACEIs), angiotensin receptor blockers (ARBs) or $\beta$-blockers alone or in combination, we compared indices of short-term BPV according to the different treatments. Short-term BPV was calculated as the standard deviation (s.d.) of $24 \mathrm{~h}$, daytime or nighttime systolic blood pressure and diastolic blood pressure (SBP and DBP). Short-term BPV was compared between patients treated with a given antihypertensive class of interest (alone or in combination) and those not treated with this class, after controlling for ambulatory average blood pressure, heart rate, age, gender, propensity scores and carotid-femoral pulse wave velocity. Patients treated with CCBs $(n=1247)$ or diuretics $(n=1486)$ alone, or in addition to other drugs had significant lower s.d. of 24-h SBP compared with those not treated with these classes (mean differences in s.d. $-0.50 \pm 0.50 \mathrm{~mm} \mathrm{Hg}, P=0.001$ and $-0.17 \pm 0.15 \mathrm{~mm} \mathrm{Hg}, P=0.05$, respectively). There was no significant difference regarding treatment with or without ARBs, ACEls and $\beta$-blockers. The combinations of CCBs with diuretics or ARBs on top of other treatments resulted in a lower 24-h SBP variability (mean differences in s.d. $-0.43 \pm 0.17 \mathrm{~mm} \mathrm{Hg}, P=0.02$ and $-0.44 \pm 0.19 \mathrm{~mm} \mathrm{Hg}, P=0.005$ vs. other combination uses, respectively). Antihypertensive drug classes have differential effects on short-term BPV with a greater reduction in patients treated with CCBs and diuretics. The combinations of CCBs with diuretics may be the most efficient treatments in lowering BPV.

Hypertension Research (2014) 37, 585-590; doi:10.1038/hr.2014.33; published online 27 March 2014

Keywords: ambulatory blood pressure monitoring; antihypertensive drug classes; arterial hypertension; arterial stiffness; blood pressure variability

\section{INTRODUCTION}

Increased short-term blood pressure variability (BPV), defined as the degree of blood pressure (BP) variations within a 24-h period, predicts organ damage ${ }^{1,2}$ and is associated with an increased risk of cardiovascular events, ${ }^{3-9}$ diabetes mellitus ${ }^{10}$ and cognitive decline. ${ }^{11,12}$

Although the possibility to obtain a reduction of cardiovascular risk by lowering BPV remains to be demonstrated, short-term BPV has been suggested as a target for antihypertensive treatment. ${ }^{13}$ Antihypertensive treatment may lead to a reduction in BPV by reducing the average BP (BPV being strongly dependent on BP levels $)^{14}$ and by improving baroreflex sensitivity, as shown by changes in heart rate variability. ${ }^{15,16}$ However, whether antihypertensive drug treatment reduces BPV independently from the concomitant reduction in mean BP levels, and whether different antihypertensive drug classes have the same impact on $\mathrm{BPV},{ }^{17-19}$ this remains to be clarified. A meta-analysis focusing not on 24-h BPV but on visit-tovisit $\mathrm{BP}$ instability reported evidence of specific drug class effects on interindividual BPV compared with placebo. ${ }^{19}$ Moreover, in hypertensive rats, the combination of different antihypertensive drug classes resulted in a greater reduction of short-term BPV compared with a single-drug regimen. ${ }^{20}$ On the other hand, limited evidence is available on the occurrence of possible differences in 24-h BPV in humans as a result of treatment with different antihypertensive drug classes, administered either as monotherapy or in combination..$^{21}$

The aim of the present study was to compare short-term BPV over $24 \mathrm{~h}$ in a cohort of hypertensive patients treated with one of the five antihypertensive classes recommended for hypertension management, that is, calcium channel blockers (CCBs), diuretics, angiotensinconverting enzyme inhibitors (ACEIs), angiotensin receptor blockers

\footnotetext{
${ }^{1}$ Department of Clinical Pharmacology, AP-HP, H.Mondor-A.Chenevier hospital, Créteil, France; ${ }^{2}$ Paris-Est University, Faculté de Médecine, Créteil, France; ${ }^{3}$ Neuropsychologie Interventionnelle, INSERM U955, Créteil, France; ${ }^{4}$ Fédération de Cardiologie, Créteil, France; ${ }^{5}$ Department of Cardiology, San Luca Hospital, Istituto Auxologico Italiano, Milan, Italy and ${ }^{6}$ Department of Health Sciences, University of Milano-Bicocca, Milan, Italy

Correspondence: Dr P Maison, Pharmacologie Clinique, CHU Henri Mondor, 51 avenue du Maréchal de Lattre de Tassigny, Créteil 94100, France. E-mail: patrick.maison@hmn.aphp.fr

Received 6 July 2013; revised 7 November 2013; accepted 15 December 2013; published online 27 March 2014
} 
(ARBs) and $\beta$-blockers, in monotherapy or by combination among them. All patients underwent 24-h ambulatory BP monitoring (ABPM) under standardized conditions during treatment.

\section{METHODS}

Treated hypertensive patients, referred to the Clinical Pharmacology Department of Henri Mondor Hospital, Créteil, France, for ABPM) to assess antihypertensive treatment efficacy from January 2005 to April 2011 were included in this cross-sectional survey. All patients underwent a thorough clinical examination, 24-h ABPM and measurement of carotid-femoral pulse wave velocity (CfPWV). Exclusion criteria were as follows: secondary causes of hypertension, treatment for heart failure, chronic renal failure, pregnancy, history of transplantation and modification of antihypertensive treatment within the last 4 weeks. The study was approved by the local ethics committee (CPP Ile de France IX, France).

Information on antihypertensive treatment was derived from medical prescriptions and compliance with prescribed treatment was checked by the physician in charge. Patients with a history of coronary artery disease, peripheral vascular disease and cerebrovascular disease were considered as having cardiovascular disease.

BP was monitored throughout $24 \mathrm{~h}$ using a non-invasive validated ambulatory auscultatory device (Diasys Integra, Novacor, Rueil-Malmaison, France), set to take a reading every $15 \mathrm{~min}$ throughout the $24 \mathrm{~h}$. Short-term BPV was calculated as the standard deviation (s.d.) of $24 \mathrm{~h}$, daytime and nighttime average systolic and diastolic BP values. Daytime and nighttime periods were defined for each patient according to their rising and sleep times, recorded in a diary. The validity of the information on wake and sleep times provided by the patient was confirmed through use of a position sensor, coupled with the ABPM device. Subjects having ABPM with $<80 \%$ of valid measurements were excluded from this analysis.

CfPWV was measured using the foot-to-foot velocity method (Complior SP, Alam Medical, Vincennes, France), as previously published and validated. ${ }^{22-24}$ The carotid-femoral straight distance was measured with a flexible tape meter over the body surface.

The representativeness of our population with regard to the issues addressed was tested by comparing the relationship between short-term BPV and previously reported determinants of BPV that is, age, gender, BP, heart rate and CfPWV with the corresponding relationship observed in other similar populations. ${ }^{6,18,25,26}$

\section{Statistical analysis}

Quantitative data were expressed as means \pm s.d. Univariate analyses were performed using $\chi^{2}$, Fisher exact test and Student's $t$-test to compare characteristics between drug class and control groups. Drug class groups were defined regardless of the number of other compounds taken by a patient in addition to the drug or drug combination of interest. According to this approach, patients treated with the antihypertensive drug class (or drug combination) of interest were compared with all other patients not treated with that drug class (or drug combination), that is, with patients taking any other antihypertensive treatments. The link between BPV and variables possibly associated with BP fluctuations was explored through Pearson' correlation coefficient.

To offset a lack of randomization due to differences in patients' treatment assignment by their physician, we calculated propensity scores (PSs), representing the conditional probability of receiving a particular treatment given a set of observed pre-treatment variables. ${ }^{27,28}$ PSs were estimated for each antihypertensive drug class by logistic regression analysis. Independent variables were the variables suspected to influence a drug class prescription that were significantly different between users and nonusers of any given treatment $(P<0.20)$. The PSs corresponded to the estimated predicted probabilities of assignment to each treatment group calculated for each subject.

We first compared the BPV parameters of patients receiving a given antihypertensive drug class of interest (regardless of the concomitant prescription of other drugs) with those of patients not treated with that class, but receiving any other type of antihypertensive drugs (control group). Logistic regression models were used for the comparison of s.d. between the different antihypertensive drug classes as defined above, with the drug class use as the independent variable, $24 \mathrm{~h}$, daytime and nighttime BP s.d. as the dependent variables (separately entered in the model to avoid collinearity problems), and ambulatory average BP ( $24 \mathrm{~h}$, day or nighttime as appropriate), heart rate, age, gender, corresponding PSs and CfPWV, as covariates. Second, we limited the analysis to patients treated with only one antihypertensive drug class. We compared the effects of each class with those of any other drug, considered one by one using pairwise comparisons in a linear mixed model, adjusted for $24-\mathrm{h}$ BP, heart rate, age, gender, corresponding PSs and CfPWV with a fixed effect for treatment and a random effect for each subject. Both types of analyses were then performed also to compare the effects of different combination therapies with two antihypertensive classes on BPV.

Two-sided $P \leqslant 0.05$ was considered statistically significant. Sample size calculation was performed based on previously published data, ${ }^{18}$ by expecting an average value of 24-h systolic BP s.d. of $12.5 \pm 3 \mathrm{~mm} \mathrm{Hg}$ under treatment. At least 99 subjects by groups were found to be necessary to highlight a difference between treatments in s.d. of 24 -h BP of $10 \%$, with a $5 \%$ $\alpha$-risk and a power of $80 \%$. Analyses were conducted using STATA 9.1 (StataCorp, College Station, TX, USA) and SPSS 17.0 (SPSS, Chicago, IL, USA).

\section{RESULTS}

The study included 2780 treated hypertensive patients. Clinical characteristics of the whole population and of each group of patients treated with a given antihypertensive drug class of interest are reported in Table $1.709(26 \%)$ patients received treatment with a single antihypertensive treatment, and 951 (34\%), 676 (24\%) and 444 (16\%) had a combination of two, three and four or more treatments, respectively. Patients with a single treatment were significantly younger than patients with combination of treatments $(58.6 \pm 12.5$ years vs. $63.2 \pm 12.2$ years; $P<0.001)$, and had less diabetes, dyslipidemia and history of cardiovascular diseases (17 vs. $31 \%$; 42 vs. $60 \%$ and 18 vs. $34 \%$, respectively; $P<0.01$ for all).

Regardless of the presence of other antihypertensive drug classes, $1486(53 \%)$ patients were treated with a diuretic, $1339(48 \%)$ with an ARB, $1292(47 \%)$ with a $\beta$-blocker, 1247 (45\%) with a CCB and 813 (29\%) with an ACEI. Characteristics of these groups and of the total population are presented in Table 1 .

Significant correlations were found between s.d. of 24-h systolic BP (SBP) and age, 24-h average SBP and CfPWV $(r=0.20, r=0.21$ and $r=0.32$, respectively, $P<0.001$ for all). s.d. of 24 -h diastolic BP (DBP) was also correlated with heart rate and 24-h average DBP ( $r=0.11$ and 0.26 , respectively; $P<0.001$ for both). Similar results were obtained for s.d. of daytime or nighttime SBP and DBP (data not shown).

Figure 1 shows the mean differences in s.d. of $24 \mathrm{~h}$, daytime and nighttime BP between patients treated with a given antihypertensive class (alone or in combination) and a control group, that is, a group defined as patients not receiving the drug under assessment. Patients treated with CCBs or diuretics alone or in addition to other drugs had significantly lower s.d. of 24-h SBP compared with those not treated with these classes (mean differences in s.d. of 24-h SBP: $\quad-0.50 \pm 0.15 \mathrm{~mm} \mathrm{Hg}$ and $-0.17 \pm 0.15 \mathrm{~mm} \mathrm{Hg}$, respectively, $P<0.001)$. In addition, SDs of $24 \mathrm{~h}$ and nighttime DBP were significantly reduced in patients treated with CCBs. No significant difference was found between patients treated with ARBs and the control group. In contrast, patients treated with ACEIs had a significantly higher s.d. of daytime SBP than controls, and those treated with a $\beta$-blocker a significantly higher s.d. of $24 \mathrm{~h}$, daytime and nighttime DBP.

In the pairwise comparisons between the 709 patients receiving a given antihypertensive therapy as monotherapy alone, the s.d. of 
Table 1 Clinical characteristics of the treated hypertensive subjects

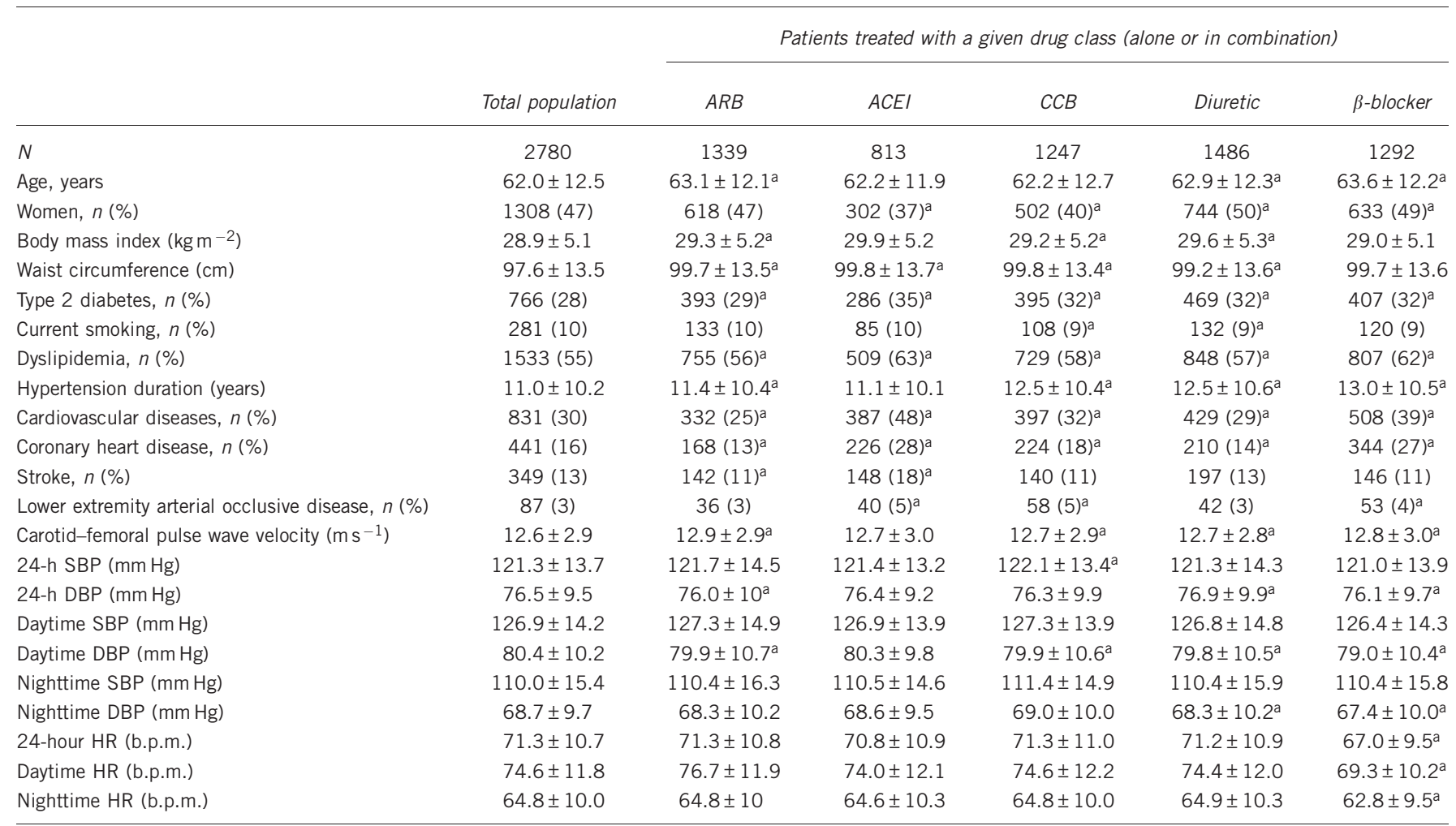

Abbreviations; b.p.m., beats per minute; DBP, diastolic blood pressure; HR, heart rate; SBP, systolic blood pressure.

aThis symbol indicates the occurrence of significant differences in the reported parameters between patients receiving a given specific drug class and a control group including all patients not receiving the drug under consideration.

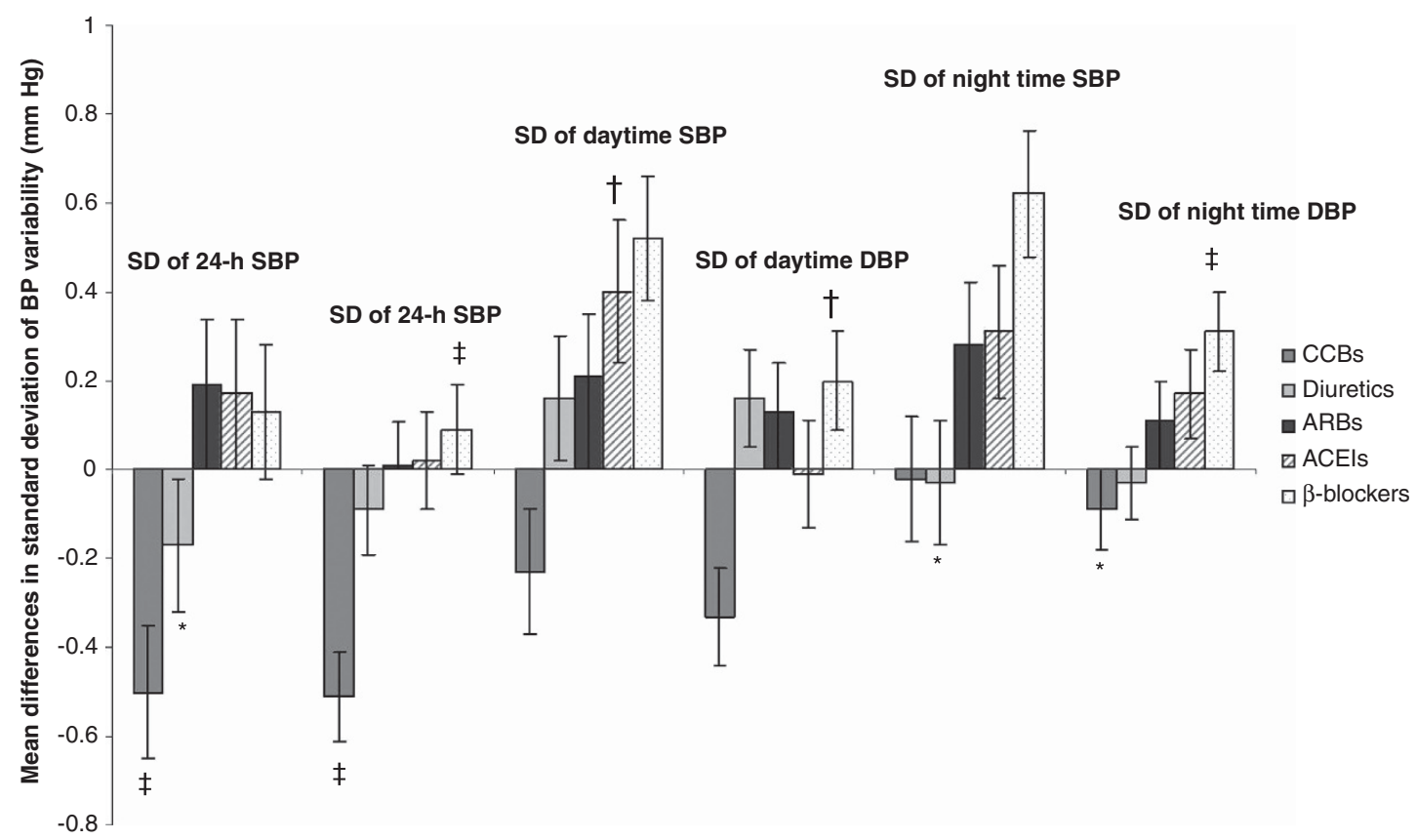

Figure 1 Mean differences \pm s.d. $(\mathrm{mm} \mathrm{Hg}$ ) in s.d. of $24 \mathrm{~h}$, daytime and nighttime BP between patients treated with a given antihypertensive class (alone or in combination) and patients not receiving the drug under assessment, but receiving any other type of antihypertensive (control group). ${ }^{*} P \leqslant 0.05$, $\dagger P \leqslant 0.01, \ddagger P \leqslant 0.001$. P-values result from logistic regression models with antihypertensive drug classes as the independent variable, short-term BPV as the dependant variable, ambulatory BP and heart rate (24-h, day or nighttime as appropriate), age, gender, carotid-femoral pulse wave velocity and the corresponding propensity score, as covariates. 
Table 2 Mean differences \pm s.d. ( $\mathrm{mm} \mathrm{Hg}$ ) in standard deviation of $24 \mathrm{~h}$, daytime and nighttime BP between patients treated with combination therapies with diuretics or calcium channel blockers and a control group, not receiving other antihypertensive treatment than the combination therapies under assessment

\begin{tabular}{|c|c|c|c|c|c|c|c|}
\hline & $\begin{array}{c}\text { CCBs + diuretics } \\
\text { vs. control } \\
n=696 / 2084\end{array}$ & $\begin{array}{c}C C B s+A R B s \\
\text { vs. control } \\
\mathrm{n}=560 / 2220\end{array}$ & $\begin{array}{c}C C B s+A C E l s \\
\text { vs. control } \\
n=342 / 2438\end{array}$ & $\begin{array}{c}\text { CCBs+ } \\
\beta \text {-blockers } \\
\text { vs. control } \\
\mathrm{n}=543 / 2237\end{array}$ & $\begin{array}{c}\text { Diuretics }+ \text { ARBs } \\
\text { vs. control } \\
\mathrm{n}=819 / 1961\end{array}$ & $\begin{array}{c}\text { Diuretics }+ \text { ACEIs } \\
\text { vs. control } \\
\mathrm{n}=410 / 2370\end{array}$ & $\begin{array}{c}\text { Diuretics }+ \\
\beta \text {-blockers } \\
\text { vs. control } \\
\mathrm{n}=737 / 2043\end{array}$ \\
\hline S.d. of 24-h SBP & $-0.43 \pm 0.17^{\dagger}$ & $-0.44 \pm 0.19^{\dagger}$ & $0.04 \pm 0.23$ & $-0.48 \pm 0.19 \ddagger$ & $0.03 \pm 0.17$ & $0.03 \pm 0.21$ & $-0.07 \pm 0.17$ \\
\hline S.d. of 24-h DBP & $-0.51 \pm 0.12^{\ddagger}$ & $-0.34 \pm 0.13$ & $-0.40 \pm 0.16$ & $-0.30 \pm 0.13$ & $0.09 \pm 0.11$ & $0.08 \pm 0.14$ & $-0.005 \pm 0.11$ \\
\hline $\begin{array}{l}\text { S.d. of daytime } \\
\text { SBP }\end{array}$ & $-0.12 \pm 0.17$ & $-0.17 \pm 0.18^{*}$ & $0.38 \pm 0.22$ & $0.06 \pm 0.18$ & $0.29 \pm 0.16$ & $0.36 \pm 0.30$ & $0.24 \pm 0.16$ \\
\hline $\begin{array}{l}\text { S.d. of daytime } \\
\text { DBP }\end{array}$ & $-0.24 \pm 0.13$ & $-0.18 \pm 0.14$ & $-0.22 \pm 0.17$ & $-0.14 \pm 0.14$ & $0.26 \pm 0.12$ & $0.08 \pm 0.15$ & $0.13 \pm 0.13$ \\
\hline $\begin{array}{l}\text { S.d. of nighttime } \\
\text { SBP }\end{array}$ & $0.13 \pm 0.16$ & $0.19 \pm 0.17$ & $0.47 \pm 0.21$ & $0.24 \pm 0.17$ & $0.19 \pm 0.15$ & $0.13 \pm 0.19$ & $0.29 \pm 0.15$ \\
\hline $\begin{array}{l}\text { S.d. of nighttime } \\
\text { DBP }\end{array}$ & $-0.11 \pm 0.10^{\dagger}$ & $-0.01 \pm 0.11$ & $0.22 \pm 0.14$ & $0.16 \pm 0.11$ & $-0.01 \pm 0.10$ & $0.12 \pm 0.13$ & $-0.26 \pm 0.10$ * \\
\hline
\end{tabular}

Abbreviations: ACEIs, angiotensin-converting enzyme inhibitors; ARBs, angiotensin receptor blockers; BP, blood pressure; BPV, blood pressure variability; CCBs, calcium channel blockers; DBP, diastolic blood pressure; SBP, systolic blood pressure.

${ }^{*} P \leqslant 0.05,{ }^{P} P \leqslant 0.01, \ddagger P \leqslant 0.001$. $P$-values result from logistic regression models with antihypertensive drug classes as the independent variable, short-term BPV as the dependant variable, ambulatory blood pressure and heart rate $(24 \mathrm{~h}$, day or nighttime as appropriate), age, gender, carotid-femoral pulse wave velocity and the corresponding propensity score, as covariates.

nighttime SBP remained lower with CCBs $(n=164)$ and diuretics $(n=53)$ but was also lower with ARBs $(n=228)$ compared with patients treated with $\beta$-blockers $(n=136)$ (mean difference in s.d.: $-1.4 \pm 0.51 \mathrm{~mm} \mathrm{Hg}, P=0.007 ;-1.4 \pm 0.71, P=0.05$ and $-1.1 \pm 0.48 \mathrm{~mm} \mathrm{Hg}, P=0.02$, respectively). No significant difference was observed when comparing patients treated with ACEIs $(n=125)$ and those treated with the other monotherapies.

Comparisons between the patients treated with a combination of two antihypertensive drug classes, alone or in addition to other drugs, and patients receiving any other treatment with the exception of the two drugs of interest, were also performed, focusing on the differences in the SDs of 24-h, daytime and nighttime SBP or DBP (Table 2). In patients treated with the combinations of CCBs with diuretics, CCBs with ARBs or CCBs with $\beta$-blockers, SDs of 24-h SBP were significantly lower compared with patients not receiving these treatments (control group). The SDs of 24-h and nighttime DBP were significantly reduced in patients treated with CCBs and diuretics. The combination of diuretics with $\beta$-blockers was also associated with a significantly lower s.d. of nighttime DBP. Finally, s.d. of daytime SBP was significantly reduced when CCBs were combined with ARBs. There were no significant differences regarding other combinations.

The pairwise comparisons between patients treated with the various possible combinations of only two antihypertensive agents confirmed the finding of a reduction in BPV in patients treated with CCBs and diuretics. This combination $(n=55)$ was associated with lower SDs of 24-h SBP and DBP compared with the effects of the eight other combinations of two antihypertensive drugs, except for the combination of CCBs with ARBs $(n=86)$ that had similar effects on s.d. of 24-h SBP. The nighttime s.d. was lower with the combination of CCBs and diuretics compared with the combination between $\mathrm{CCBs}$ and ARBs $(-1.3 \pm 0.42 \mathrm{mmHg}, P=0.002)$. The combination of CCBs and ARBs was associated with a significantly lower s.d. of 24 -h DBP $(-0.96 \pm 0.39 \mathrm{~mm} \mathrm{Hg}, P=0.01)$ when compared with the effect of a combination between ACEIs and diuretics $(n=93)$. There were no significant differences for the other combinations in this sub-group.

\section{DISCUSSION}

Results of the present study indicate that antihypertensive drug classes may have different effects on short-term BP variability in a daily life clinical setting, independently from the effects of major confounders such as BP level, age, gender, heart rate, CfPWV and factors suspected to influence the choice of antihypertensive drug classes' prescription. In particular, we found that the use of CCBs and, to a lesser extent, diuretics, was associated with a lower short-term BPV compared with the other classes (ARBs, ACEIs and $\beta$-blockers). Also, the association of CCBs with diuretics was associated with a lower short-term BPV compared with other combination of antihypertensive classes.

Our findings of a lower short-term BPV with CCBs is in accordance with previous observational and experimental studies. ${ }^{6,9,17}$ The X-CELLENT trial showed that CCBs and diuretics, but not ARBs, lead to a decrease of 24-h BPV compared with placebo. ${ }^{18}$ In another randomized trial including untreated hypertensive patients, long-term treatment with CCBs significantly decreased the $24-\mathrm{h}$ BPV, in contrast to ARBs that did not affect BPV. ${ }^{17}$ Finally, a study in hypertensive patients with diabetes showed that treatment with the CCB Lacidipine was able to significantly reduce 24-h BPV (assessed through analysis of beat-by-beat $24 \mathrm{~h}$ ambulatory BP recordings) after adjustment on $\mathrm{BP}$ lowering, determining at the same time an improvement in spontaneous cardiac baroreflex sensitivity. ${ }^{21}$

A recent meta-analysis of randomized trials ${ }^{19}$ concluded for a reduction of the visit-to-visit variability in SBP, that is, fluctuations of SBP over long periods of observation, with the use of CCBs and non-loop diuretics. In contrast, the use of ARBs, $\beta$-blockers and ACEIs was associated with an increase of the visit-to-visit variability in SBP. The ASCOT-BPLA trial also showed opposite effects of $\beta$ blockers and $\mathrm{CCBs}$ on within-individual variability in $\mathrm{BP}$ independently of their effects on $\mathrm{BP}^{29}$ Our results, obtained from real-life conditions of daily practice, in contrast with randomized clinical trial conditions, confirm the differences of antihypertensive drugs in their effects on short-term BPV, and provide additional information by showing that patients treated with CCBs had a lower 
BPV than patients not treated with CCBs, independently from the effects of other concomitant antihypertensive drugs.

Our results regarding combinations of antihypertensive drugs suggest that the combination of CCBs and diuretics or CCBs and ARBs could provide a lower 24-h, daytime or nighttime BPV compared with other combinations, regardless of the total number of drugs administered. These results were confirmed in pairwise comparisons between combinations of two different antihypertensive drug classes, and are in accordance with recent results on day-by-day BPV. ${ }^{30}$ Benefits of specific combination therapies on visit-to-visit BP variability had also been suggested by Webb's meta-analysis ${ }^{31}$ finding that the reduction of interindividual systolic BPV with CCBs and diuretics was maintained when these drugs were used in combination with other drug classes. The authors also showed that adding a CCB or a diuretic to any antihypertensive treatment reduced the interindividual variability to the same extent as seen with monotherapy of these drugs vs. placebo. In contrast, they showed that adding an ACEI or an ARB agent to another class had no significant impact. Similar results were found in another study showing that the addition of CCBs decreased the systolic and diastolic 24-h BPV in hypertensive patients treated with ARBs. ${ }^{32}$ Our results expand these findings by demonstrating benefits of these drugs on short-term BPV as well, and in the real-life conditions of daily practice, in contrast with clinical trial protocol conditions.

Given the well-known relation between mean BP and BPV, it might be argued that the different reduction in BPV observed with the different drug classes might simply reflect different mean BP lowering levels. ${ }^{33,34}$ However, the persistence of these differences after accounting for the impact of changes in mean BP levels in our multivariate analysis models seems to exclude the possibility that different degrees of mean BP changes between antihypertensive drug classes might be the main explanation for the observed different effects on BPV.

BPV has been related to arterial stiffness ${ }^{17,26,35}$ and different antihypertensive drug classes have shown contrasting effects on arterial stiffness. ${ }^{36}$ Although the mechanisms underlying the differences in BPV between the antihypertensive drug classes are uncertain, changes in peripheral vascular distensibility might thus have a role. ${ }^{37} \quad \beta$-blockers reduce arterial compliance via vasoconstrictive effects, whereas CCBs have vasodilating properties that may in contrast result in increasing arterial compliance. In addition, the reduced 24-h BPV with CCBs treatment has been associated with a reduction in PWV. ${ }^{17}$ Thus, it supports the hypothesis of a clinically relevant association between changes in BPV and changes in arterial stiffness. ${ }^{25}$ Improvement of arterial distensibility has been indeed suggested as a possible mechanism for the reduction in BPV by some antihypertensive agents. ${ }^{38}$ In our treated hypertensive patients, there was a positive relationship between BPV and CfPWV. However, the cross-sectional design of our study does not allow concluding for the occurrence of a cause-effect link between changes in BPV and changes in arterial stiffness, leaving the possibility open, however, that a reduction in arterial stiffness might be either the cause and/or the effect of a reduction in BPV by treatment.

Regarding the representativeness of our population, some of our findings are in line with those previously found in other similar populations. As an example, in our subjects, as shown in previous studies, BPV was related to age, sex and BP level, all these parameters having already been identified as significant determinants of BPV. ${ }^{6,18,25}$ The representativeness of our study population is also supported by the finding that the rates of use of the different drugs administered in our patients were consistent with the ones reported by the French National Health Insurance Fund for Salaried Workers' study in 2006 (comprising a representative sample of the French population), the percentage of drug prescription being $54.7 \%$ for diuretics, $28.7 \%$ for ACEIs, $38.9 \%$ for $\beta$-blockers, $36.7 \%$ for ARBs and $29 \%$ for CCBs. ${ }^{39}$ The percentages of utilization for each drug class were also within the range reported for European countries and for the United States of America. ${ }^{40}$

We also have to acknowledge a few limitations of our study, however. First, the present study has a cross-sectional design, and the patients included were not randomized to the different drug classes. Yet, we have reduced the possible drawbacks of such a limitation, by using PSs, which, although unable to completely substitute randomization, allowed us to overcome a selection bias due to possible noncasual differences in patients' treatment assignment. Second, in the pairwise comparisons of the single-drug classes, significant differences were observed only for nighttime BPV, that was lower with CCBs compared with $\beta$-blockers and ACEIs. The limited sample size of these last comparisons may have underestimated significant results for 24-h or daytime BPV. Third, the appropriateness of s.d. as an index of BP variability has been recently questioned, in particular as it could be influenced by nocturnal BP. ${ }^{41}$

However, these limitations should also be considered in the perspective of the multiple strengths of our study. First, to the best of our knowledge, this is the first study that directly compares the effect of the five main antihypertensive drug classes on short-term BPV in a large group of 'real-life' hypertensive patients. Second, the different effects observed between the different drug classes were independent of the concomitant changes in mean $\mathrm{BP}$, and the major determinants of BPV (heart rate, age, gender and CfPWV). ${ }^{18,25,26,38}$ Third, we assessed BPV by using the s.d. of 24-h average BP. This was done because the association of target-organ damage, cardiovascular events and mortality with BPV was frequently assessed in various population-based studies through the quantification of the s.d. of $24-\mathrm{h} \mathrm{BP}{ }^{3,4,42-45}$ The s.d. of 24 -h average BP, however, has been shown to include not only the contribution of fast, short-term BP fluctuations, but also the contribution of day-night BP changes, the latter carrying a favourable rather than an adverse prognostic impact, at variance from short-term BP variations. ${ }^{41}$ In our analysis, however, we have also separately computed the s.d. of daytime and nighttime SBP and DBP to prevent our results being undetermined by these methodological issues. Indeed, in the ASCOT-BPLA study, daytime BP variability, expressed as s.d. of daytime SBP, correlated with visit-to-visit variability, and predicted vascular events independently of daytime average SBP. ${ }^{29}$ Yet nighttime BPV was considered to be a more pronounced cardiovascular risk factor than daytime BPV. ${ }^{7,44}$

Our results, for the first time, demonstrate that different antihypertensive drug classes have different effects on short-term BPV in the real-life conditions of daily practice, with a more evident reduction in short-term BP variability when treatment is based on $\mathrm{CCBs}$ and diuretics, alone or in combination with other antihypertensive classes. The reliability of our results should be demonstrated using new indices of variability (such as average real variability), and the clinical relevance of these statistical differences in BPV between different drug classes deserves further investigations through longitudinal, randomized intervention trials. These trials should be aimed at exploring whether the benefits of BPV lowering by CCBs and diuretics might translate into a cardiovascular risk reduction in humans. Indeed, until now, the benefits of treatment-induced reduction in BPV have been related to a reduction in end-organ damage only in the setting of experimental animal studies. ${ }^{20,46}$ 


\section{CONFLICT OF INTEREST}

The authors declare no conflict of interest.

\section{ACKNOWLEDGEMENTS}

NLM received a grant from the Fondation pour la Recherche Medicale and the Fondation des Treilles.

1 Parati G, Pomidossi G, Albini F, Malaspina D, Mancia G. Relationship of 24-hour blood pressure mean and variability to severity of target-organ damage in hypertension. J Hypertens 1987; 5: 93-98.

2 Frattola A, Parati G, Cuspidi C, Albini F, Mancia G. Prognostic value of 24-hour blood pressure variability. J Hypertens 1993; 11: 1133-1137.

3 Mancia G, Parati G, Hennig M, Flatau B, Omboni S, Glavina F, Costa B, Scherz R Bond G, Zanchetti A. ELSA investigators. Relation between blood pressure variability and carotid artery damage in hypertension: baseline data from the European Lacidipine Study on Atherosclerosis (ELSA). J Hypertens 2001; 19: 1981-1989.

4 Kikuya M, Hozawa A, Ohokubo T, Tsuji I, Michimata M, Matsubara M, Ota M, Nagai K, Araki T, Satoh H, Ito S, Hisamichi S, Imai Y. Prognostic significance of blood pressur and heart rate variabilities: the Ohasama study. Hypertension 2000; 36: 901-906.

5 Sander D, Kukla C, Klingelhofer J, Winbeck K, Conrad B. Relationship between circadian blood pressure patterns and progression of early carotid atherosclerosis: a 3 year follow-up study. Circulation 2000; 102: 1536-1541.

6 Pringle E, Phillips C, Thijs L, Davidson C, Staessen JA, de Leeuw PW, Jaaskivi M, Nachev C, Parati G, O'Brien ET, Tuomilehto J, Webster J, Bulpitt CJ, Fagard RH. SystEur investigators. Systolic blood pressure variability as a risk factor for stroke and cardiovascular mortality in the elderly hypertensive population. J Hypertens 2003; 21 2251-2257.

7 Ozawa M, Tamura K, Okano Y, Matsushita K, Ikeya Y, Masuda S, Wakui H, Dejima T, Shigenaga A, Azuma K, Ishigami T, Toya Y, Ishikawa T, Umemura S. Identification of an increased short-term blood pressure variability on ambulatory blood pressure monitoring as a coronary risk factor in diabetic hypertensives. Clin Exp Hypertens 2009; 31 259-270.

8 Palatini P, Penzo M, Racioppa A, Zugno E, Guzzardi G, Anaclerio M, Pessina AC. Clinical relevance of nighttime blood pressure and of daytime blood pressure variability. Arch Intern Med 1992; 152: 1855-1860.

9 Mancia G, Parati G, Bilo G, Maronati A, Omboni S, Baurecht H, Hennig M, Zanchetti A Assessment of long-term antihypertensive treatment by clinic and ambulatory blood pressure: data from the European Lacidipine Study on Atherosclerosis. J Hypertens 2007; 25: 1087-1094.

10 Ozawa M, Tamura K, Iwatsubo K, Matsushita K, Sakai M, Tsurumi-Ikeya Y, Azuma K, Shigenaga A, Okano Y, Masuda S, Wakui H, Ishigami T, Umemura S. Ambulatory blood pressure variability is increased in diabetic hypertensives. Clin Exp Hypertens 2008 30: 213-224

11 Bellelli G, Pezzini A, Bianchetti A, Trabucchi M. Increased blood pressure variability may be associated with cognitive decline in hypertensive elderly subjects with no dementia. Arch Intern Med 2002; 162: 483-484.

12 Sakakura K, Ishikawa J, Okuno M, Shimada K, Kario K. Exaggerated ambulatory blood pressure variability is associated with cognitive dysfunction in the very elderly and quality of life in the younger elderly. Am J Hypertens 2007; 20: 720-727.

13 Schillaci G, Pucci G, Parati G. Blood pressure variability: an additional target for antihypertensive treatment? Hypertension 2011; 58: 133-135.

14 Mancia G, Ferrari A, Gregorini L, Parati G, Pomidossi G, Bertinieri G, Grassi G, di Rienzo M, Pedotti A, Zanchetti A. Blood pressure and heart rate variabilities in normotensive and hypertensive human beings. Circ Res 1983; 53: 96-104.

15 Floras JS, Hassan MO, Jones JV, Osikowska BA, Sever PS, Sleight P. Factors influencing blood pressure and heart rate variability in hypertensive humans. Hypertension 1988; 11: 273-281.

16 Mancia G, Parati G, Pomidossi G, Casadei R, Di Rienzo M, Zanchetti A. Arterial baroreflexes and blood pressure and heart rate variabilities in humans. Hypertension 1986; 8: 147-153

17 Ichihara A, Kaneshiro Y, Takemitsu T, Sakoda M. Effects of amlodipine and valsartan on vascular damage and ambulatory blood pressure in untreated hypertensive patients. J Hum Hypertens 2006; 20: 787-794.

18 Zhang Y, Agnoletti D, Safar ME, Blacher J. Effect of antihypertensive agents on blood pressure variability: the Natrilix SR versus candesartan and amlodipine in the reduction of systolic blood pressure in hypertensive patients (X-CELLENT) study. Hypertension 2011; 58: 155-160.

19 Webb AJ, Fischer U, Mehta Z, Rothwell PM. Effects of antihypertensive-drug class on interindividual variation in blood pressure and risk of stroke: a systematic review and meta-analysis. Lancet 2010; 375: 906-915.

$20 \mathrm{Xie} \mathrm{HH}$, Miao CY, Jiang YY, Su DF. Synergism of atenolol and nitrendipine on hemodynamic amelioration and organ protection in hypertensive rats. J Hypertens 2005; 23: 193-201.

21 Frattola A, Parati G, Castiglioni P, Paleari F, Ulian L, Rovaris G, Mauri G, Di Rienzo M, Mancia G. Lacidipine and blood pressure variability in diabetic hypertensive patients. Hypertension 2000; 36: 622-628.
22 Asmar R, Benetos A, Topouchian J, Laurent P, Pannier B, Brisac AM, Target R, Levy BI. Assessment of arterial distensibility by automatic pulse wave velocity measurement. Validation and clinical application studies. Hypertension 1995; 26: 485-490.

23 Pereira T, Maldonado J. Comparative study of two generations of the Complior device for aortic pulse wave velocity measurements. Blood Press Monit 2010; 15: 316-321.

24 Asmar RG, London GM, O'Rourke ME, Safar ME. Improvement in blood pressure, arterial stiffness and wave reflections with a very-low-dose perindopril/indapamide combination in hypertensive patient: a comparison with atenolol. Hypertension 2001 38: 922-926.

25 Imai Y, Aihara A, Ohkubo T, Nagai K, Tsuji I, Minami N, Satoh H, Hisamichi S. Factors that affect blood pressure variability. A community-based study in Ohasama, Japan Am J Hypertens 1997; 10: 1281-1289.

26 Schillaci G, Bilo G, Pucci G, Laurent S, Macquin-Mavier I, Boutouyrie P, Battista F, Settimi L, Desamericq G, Dolbeau G, Faini A, Salvi P, Mannarino E, Parati G. Relationship between short-term blood pressure variability and large-artery stiffness in human hypertension: findings from 2 large databases. J Hypertens 2012; 60 369-377

27 Imbens GW. The role of the propensity socre in estimating dose-response functions Biometrika 2000; 87: 706-710.

28 Spreeuwenberg MD, Bartak A, Croon MA, Hagenaars JA, Busschbach JJ, Andrea H, Twisk J, Stijnen T. The multiple propensity score as control for bias in the comparison of more than two treatment arms: an introduction from a case study in mental health. Med Care 2010; 48: 166-174.

29 Rothwell PM, Howard SC, Dolan E, O'Brien E, Dobson JE, Dahlöf B, Poulter NR, Sever PS. ASCOT-BPLA and MRC Trial Investigators. Effects of beta blockers and calcium-channel blockers on within-individual variability in blood pressure and risk of stroke. Lancet Neurol 2010; 9: 469-480.

30 Parati G, Bilo G. Calcium antagonist added to angiotensin receptor blocker: a recipe for reducing blood pressure variability?: evidence from day-by-day home blood pressure monitoring. Hypertension 2012; 59: 1091-1093.

31 Webb AJ, Rothwell PM. Effect of dose and combination of antihypertensives on interindividual blood pressure variability: a systematic review. Stroke 2011; 42 2860-2865.

32 Ichihara A, Kaneshiro Y, Sakoda M, Takemitsu T, Itoh H. Add-on amlodipine improves arterial function and structure in hypertensive patients treated with an angiotensin receptor blocker. J Cardiovasc Pharmacol 2007; 49: 161-166.

33 Parati G, Faini A, Valentini M. Blood pressure variability: its measurement and significance in hypertension. Curr Hypertens Rep 2006; 8: 199-204.

34 Mancia G, Omboni S, Ravogli A, Parati G, Zanchetti A. Ambulatory blood pressure monitoring in the evaluation of antihypertensive treatment: additional information from a large data base. Blood Press 1995; 4: 148-156.

35 Schillaci G, Pucci G, Pirro M, Settimi L, Hijazi R, Franklin SS, Mannarino E. Combined effects of office and 24-h blood pressure on aortic stiffness in human hypertension. J Hypertens 2011; 29: 869-875.

36 Ong KT, Delerme S, Pannier B, Safar ME, Benetos A, Laurent S, Boutouyrie P Investigators. Aortic stiffness is reduced beyond blood pressure lowering by short-term and long-term antihypertensive treatment: a meta-analysis of individual data in 294 patients. J Hypertens 2011; 29: 1034-1042.

37 Rothwell PM. Limitations of the usual blood-pressure hypothesis and importance of variability, instability, and episodic hypertension. Lancet 2010; 375: 938-948.

38 Lacolley P, Bezie Y, Girerd X, Challande P, Benetos A, Boutouyrie P, Ghodsi N, Lucet B, Azoui R, Laurent S. Aortic distensibility and structural changes in sinoaorticdenervated rats. Hypertension 1995; 26: 337-340.

39 Samson S, Ricordeau P, Pépin A, Tilly B, Weill A, Allemand H. Hypertension artérielle et facteur de risques associés: évolutions des traitements entre 2000 et 2006 . Points de repère 2007; 10: 1-8.

40 Wang YR, Alexander GC, Stafford RS. Outpatient hypertension treatment, treatment intensification, and control in Western Europe and the United States. Arch Intern Med 2007; 167: 141-147.

41 Bilo G, Giglio A, Styczkiewicz K, Caldara G, Maronati A, Kawecka-Jaszcz K, Mancia G, Parati G. A new method for assessing 24-h blood pressure variability after excluding the contribution of nocturnal blood pressure fall. J Hypertens 2007; 25 . 2058-2066.

42 Sega R, Corrao G, Bombelli M, Beltrame L, Facchetti R, Grassi G, Ferrario M, Mancia G. Blood pressure variability and organ damage in a general population: results from the PAMELA study (Pressioni Arteriose Monitorate E Loro Associazioni). Hypertension 2002; 39: 710-714.

43 Bjorklund K, Lind L, Zethelius B, Berglund L, Lithell H. Prognostic significance of 24-h ambulatory blood pressure characteristics for cardiovascular morbidity in a population of elderly men. J Hypertens 2004; 22: 1691-1697.

44 Eguchi K, Ishikawa J, Hoshide S, Ishikawa S, Shimada K, Kario KJapan Morning Surge-Home Blood Pressure Study Investigators Group. Nighttime blood pressure variability is a strong predictor for cardiovascular events in patients with type 2 diabetes. Am J Hypertens 2009; 22: 46-51.

45 Brunelli SM, Thadhani RI, Lynch KE, Ankers ED, Joffe MM, Boston R, Chang Y, Feldman $\mathrm{HI}$. Association between long-term blood pressure variability and mortality among incident hemodialysis patients. Am J Kidney Dis 2008; 52: 716-726.

46 Liu JG, Xu LP, Chu ZX, Miao CY, Su DF. Contribution of blood pressure variability to the effect of nitrendipine on end-organ damage in spontaneously hypertensive rats. J Hypertens 2003; 21: 1961-1967. 